\title{
Experimental characterisation of fatigue crack growth based on the CTOD measured from crack tip displacement fields using DIC
}

\author{
J.M. Vasco-Olmo, F.A. Díaz \\ University of Jaén, Spain \\ jvasco@ujaen.es \\ fdiaz@ujaen.es
}

F.V. Antunes

University of Coimbra, Portugal

Fernando.ventura@dem.up.pt

M.N. James

University of Plymouth, UK

M.James@plymouth.ac.uk.

\begin{abstract}
The current work presents an experimental study on the use of the crack tip opening displacement (CTOD) to evaluate its ability to characterise fatigue crack growth. A methodology is developed to measure and to analyse the CTOD from experimental data. The vertical displacements obtained by implementing digital image correlation (DIC) on growing fatigue cracks are used to measure the CTOD. Two fatigue tests at stress ratios of 0.1 and 0.6 were conducted on compact tension (CT) specimens manufactured from a $1 \mathrm{~mm}$ thick sheet of commercially pure titanium. A sensitivity analysis to explore the effect of the position selected behind the crack tip for the CTOD measurement was performed. The analysis of a full loading cycle allowed identifying the elastic and plastic components of the CTOD. The plastic CTOD was found to be directly related to the nonlinear zone (i.e., plastic deformation) generated at the crack tip during fatigue propagation. Moreover, a linear relationship between $d a / d N$ and $\Delta \mathrm{CTOD} p$ independent of the stress ratio was found. Results show that the CTOD can be used as a viable alternative to the stress intensity factor range $(\Delta K)$ in characterising fatigue crack propagation since the parameter considers the fatigue threshold and crack shielding in an intrinsic way.
\end{abstract}

KEYWORDS. Crack tip opening displacement (CTOD); fatigue crack growth rate; DIC; range of plastic CTOD.

\section{OPEN ACCESS}

Citation: Vasco-Olmo, J.M., Díaz, F.A., Antunes, F.V., James, M.N., Experimental characterisation of fatigue crack growth based on the CTOD measured from crack tip displacement fields using DIC, Frattura ed Integrità Strutturale, 49 (2019) 658-666.

Received: 11.03 .2019

Accepted: 23.04.2019

Published: 01.07.2019

Copyright: (C) 2019 This is an open access article under the terms of the CC-BY 4.0, which permits unrestricted use, distribution, and reproduction in any medium, provided the original author and source are credited. 


\section{INTRODUCTION}

$\mathrm{F}$ atigue crack growth has been traditionally characterised by the Paris law [1], that relates the crack growth per cycle, $d a / d N$ to the stress intensity factor range, $\Delta K$. However, there are several controversial issues and unanswered questions in this field. The procedures for analysing constant amplitude fatigue under small-scale yielding conditions are well established, although a number of uncertainties remain. Variable amplitude loading, large-scale plasticity, and short cracks introduce additional complications that are not fully understood. In addition, in many materials, it is virtually impossible to characterise the fracture behaviour with linear elastic fracture mechanics (LEFM), and an alternative fracture mechanics model is required. Elastic-plastic fracture mechanics (EPFM) is applied to materials that exhibit nonlinear behaviour (i.e., plastic deformation). Hence in authors' view, the linear elastic $\Delta K$ parameter should be replaced by nonlinear crack tip parameter since fatigue crack growth is governed by nonlinear processes at the crack tip. Two elastic-plastic parameters have been proposed to be related with crack tip plastic deformation, the crack tip opening displacement (CTOD) and the $J$ contour integral. Both parameters describe crack tip conditions in elastic-plastic materials, and they can be used as a fracture criterion. CTOD is a local parameter, while the $J$ integral is used as a global criterion based on the quasi-strain energy release rate. Critical values of CTOD or $J$ give nearly size-independent measurements of fracture toughness, even for relatively large amounts of crack tip plasticity. There are limits to the applicability of these parameters but they are much less restrictive than the validity requirements of LEFM. In this work, CTOD is the parameter used to characterise fatigue crack growth.

CTOD was first observed by Wells [2] when he was attempting to measure $K_{I C}$ values in a number of structural steel. Wells found that these materials were too tough to be characterised by LEFM. While examining fractured test specimens, Wells noticed that the crack faces had moved apart prior to fracture; plastic deformation had blunted an initially sharp crack, resulting in a finite displacement at the crack tip. The degree of crack blunting increased in proportion to the toughness of the material. This observation led Wells to propose the opening at the crack tip as a measurement of fracture toughness. Nowadays, CTOD is a classical parameter in elastic-plastic fracture mechanics and it has a great importance for fatigue analysis. Crack tip blunting at maximum load and the crack tip re-sharpening at minimum load were used to explain fatigue crack growth [3]. CTOD has been experimentally measured by using extensometers located remotely to the crack tip. Thus, in compact tension (CT) specimens an extensometer with blades is located at the mouth of the specimen notch to measure the opening of the specimen [4]. In the case of middle tension (MT) specimens a pin extensometer is placed at the centre of the specimen by fixing it in two small holes [5]. Recently, full field optical techniques have become very popular for the analysis of structural integrity problems. Among them Digital Image Correlation (DIC) technique can be considered because the displacement fields at the vicinity of the crack tip can be measured with high level of accuracy [6]. Thus, in this work DIC is implemented to measure the CTOD from the relative displacement between both crack flanks. Moreover, finite element analysis has also been used to measure the CTOD numerically. The displacement at the first node behind the crack tip is generally used as an operational CTOD [7].

In a previous work, Antunes et al. [8] developed a numerical study to quantify the CTOD in a MT specimen for two aluminium alloys in order to analyse the applicability of this parameter to characterise fatigue crack growth. A relationship was found between $d a / d N$ and the plastic CTOD range for the 6082-T6 aluminium alloy independent of stress ratio, showing that the CTOD can be a viable alternative to $\Delta K$ in the analysis of fatigue crack propagation. Thus, in the current work an experimental study of the CTOD is performed by implementing the procedure developed by Antunes et al. [8] to evaluate the ability of this parameter to characterise fatigue crack growth. Note that the values of $\triangle \mathrm{CTOD}_{p}$ reported in [8] are relatively small, lower than $1 \mu \mathrm{m}$, which is certainly a challenge for the experimental determination of plastic CTOD since some controversy exists about if DIC is able to provide extremely high spatial resolution characterisation of crack tip deformation fields, including crack opening profiles [9]. The vertical displacements measured by DIC on growing fatigue cracks are used to measure the CTOD as the relative displacement existing between the crack flanks. Two fatigue tests at stress ratios of 0.1 and 0.6 were conducted on titanium CT specimens. From the analysis of a full loading cycle, the elastic and plastic CTOD could be identified. A linear relationship between $d a / d N$ and the plastic CTOD was found for both tests, showing therefore that the CTOD can be used as a viable alternative in characterising fatigue crack growth. With this work, the authors intend to contribute to a better understanding of the different mechanisms driving fatigue crack propagation and to address the outstanding controversy associated with plasticity-induced fatigue crack closure. 


\section{EXPERIMENTAL DETAILS}

wo CT specimens (dimensions shown in Fig. 1a) were manufactured from a $1 \mathrm{~mm}$ thick sheet of commercially pure Grade 2 titanium and tested in constant amplitude fatigue loading with a maximum load of $750 \mathrm{~N}$ at stress ratio values of 0.1 and 0.6 .
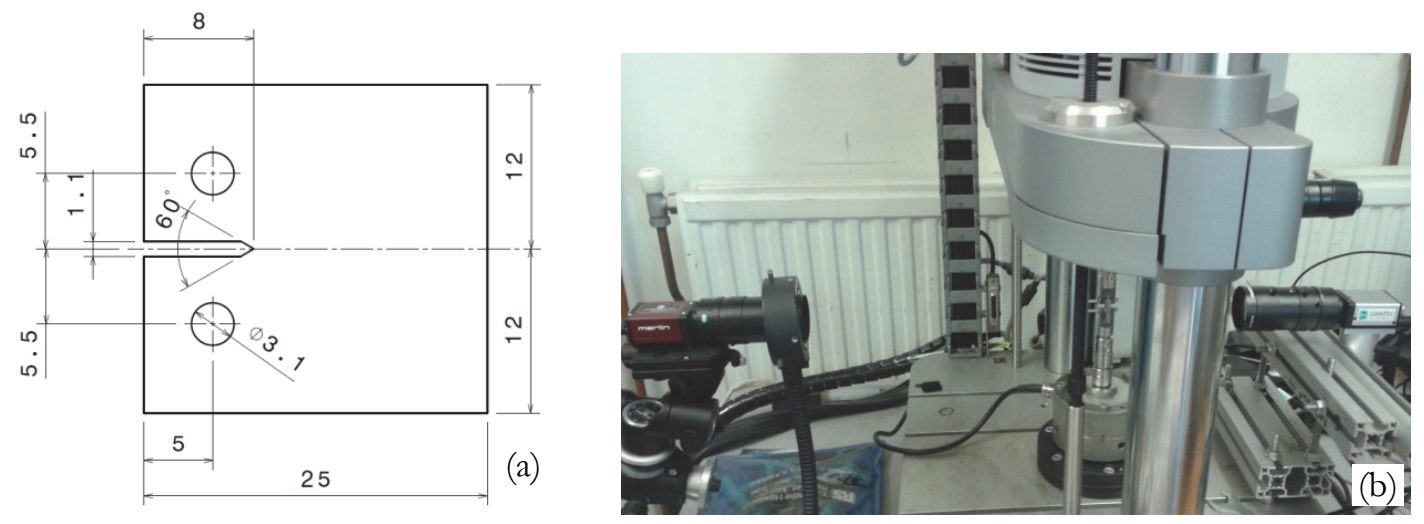

Figure 1: (a) Dimensions ( $\mathrm{mm}$ ) of the CT specimens tested. (b) Experimental set-up used for fatigue testing and data acquisition.

The two surfaces of each specimen were treated using different methods to enable simultaneous measurements of the displacement field by digital image correlation (DIC) on one side and crack length on the other. The surface used for the DIC study was sprayed with a random black speckle pattern over a white background, while the other surface of the specimen was ground and polished to allow tracking of the crack tip position with a macro-zoom lens (MLH-10X EO). Fatigue tests were conducted on an Electropuls E3000 electrodynamic machine (Fig. 1b) at a loading frequency of $10 \mathrm{~Hz}$. A CCD camera, fitted with a macro-zoom lens similar to that indicated above to increase the spatial resolution at the region around the crack tip, was placed perpendicularly to each face of the specimen. During fatigue testing, the cyclic loading was periodically paused to allow acquisition of a sequence of images at uniform increments through a complete loading and unloading cycle. The CCD camera viewing the speckled surface of the specimen was set up so that the field of view was $17.3 \times 13 \mathrm{~mm}$ (resolution of $13.7 \mu \mathrm{m} /$ pixel) with the crack path located at the centre of the image. A fibre optic ring placed around the zoom lens provided illumination of the specimen surface (also shown in Fig. 1b).

\section{METHODOLOGY FOR LOCATING THE CRACK TIP}

A $\mathrm{s}$ indicated above, CTOD is a parameter that measures the opening at the crack tip, hence the vertical displacements obtained from experiments are used in analysing its value. Examples of DIC horizontal and vertical displacement maps for a crack length of $9.40 \mathrm{~mm}$ and a load level of $750 \mathrm{~N}$ are shown in Fig. 2.

The method of obtaining the CTOD from measurements of the relative displacement between the crack flanks of selected points behind the crack tip is explained below. It is important to note that the accuracy of the results is strongly influenced by location of the assumed crack tip; accurate location is therefore important and this is found in the following manner. Firstly, the $y$-coordinate of the crack tip is found by plotting a set of profiles of vertical displacement perpendicular to the crack plane as all profiles converge onto a single point where they cross the crack plane. This is clearly seen in Fig. $3 \mathrm{a}$ and the intersection point identifies the location of the crack tip in the $y$-direction. The corresponding vertical displacement $y$ coordinate for this point is marked in Fig. 3 a because it is then used to find the $x$-coordinate of the crack tip. This is done by plotting a displacement profile in the $x$-direction, parallel with the crack direction, and locating the point that corresponds with the same displacement value $(v=0.158 \mathrm{~mm})$ previously found for the y-coordinate (Fig. $3 \mathrm{~b})$. Using this procedure, the crack tip was identified as located at the point with coordinates $x=470$ pixels and $y=468$ pixels, with the coordinate origin being at the upper left corner of the vertical displacement map (Fig. 2b).

Once the crack tip location is established, the CTOD can be obtained by defining a suitable pair of measurement points behind the crack tip, and determining the CTOD through a complete loading cycle by analysing both the loading and unloading half cycles. From this information, the portion of the cycle during which the crack is nominally closed or open 
can be found. Finally, from analysis of the portion of the load cycle during which the crack is open, both the elastic and plastic components of the CTOD can be estimated from the variation in slope observed in the CTOD versus load curves.
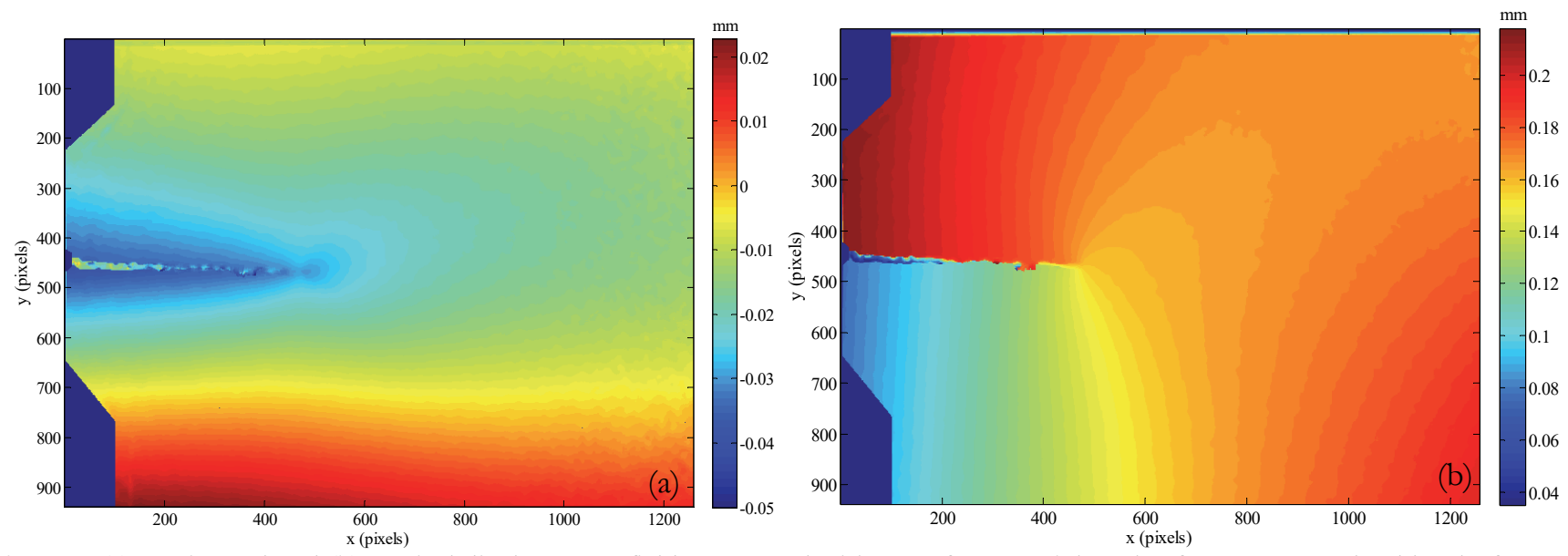

Figure 2: (a) Horizontal and (b) vertical displacement fields measured with DIC for a crack length of $9.40 \mathrm{~mm}$ at a load level of $750 \mathrm{~N}$.
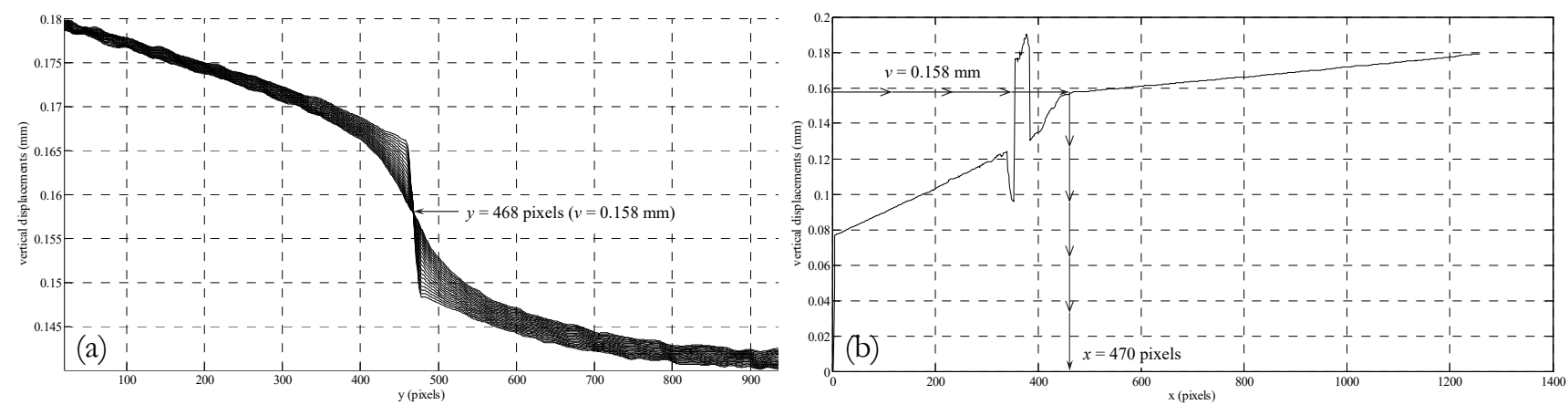

Figure 3: Graphs to support the explanation given in the paper of the methodology used for locating the crack tip: (a) $y$-coordinate and (b) $x$-coordinate.

\section{EFFECT OF THE POSITION BEHIND THE CRACK TIP}

A sensitivity analysis was performed to explore the influence of the distance behind the crack tip of the pair of points selected for the CTOD measurements, as this is clearly critical to interpreting CTOD data and correlating it with fatigue crack growth rate. The position of these two points behind the crack tip is given in Fig. 4 as $L_{x}$ in the direction of the crack plane and $L_{y}$ in the direction perpendicular to the crack. The coordinate axes have been modified establishing their origin at the coordinates found in the previous section for the crack tip.

The sensitivity analysis involved measuring the CTOD at the maximum load as a function of one of the parameters, whilst maintaining the other one fixed. Fig. 5 presents the results of this analysis, with Fig. $5 \mathrm{a}$ plotting the CTOD as a function of $L_{x}$ for various value of $L_{y}$ and Fig. $5 b$ giving it as a function of $L_{y}$ for different values of $L_{x}$. As would be expected, CTOD values increase steadily with increasing distance behind the crack tip. The key observation from these data considering Fig. 5a is that for any particular $L_{y}$ value $>10$ pixels $(136.9 \mu \mathrm{m})$ the CTOD reaches an upper bound limit for $L_{x}$ distances behind the crack tip approximately $\geq 120 \mu \mathrm{m}$. This is more clearly shown in Fig. $5 \mathrm{~b}$ where for $L_{x}$ values approximately $>8$ pixels $(82.1 \mu \mathrm{m})$ the CTOD value attains a plateau at a $L$, distance of approximately $140 \mu \mathrm{m}$. This stable plateau region is the result of rigid body motion and indicates the boundary of the region undergoing crack tip deformation and its onset can hence be used to characterise the CTOD. The plateau region is indicated with the rectangle in Fig. $5 \mathrm{~b}$ and encloses the CTOD values corresponding to the ranges 5-15 pixels $(68.4-205.3 \mu \mathrm{m})$ for $L_{x}$ and 10-15 pixels $(136.8-205.3 \mu \mathrm{m})$ for $L_{y}$. This analysis demonstrates that the CTOD is accurately characterised by using data corresponding with the position of two points with a horizontal position $L_{x}=5$ pixels $(68.4 \mu \mathrm{m})$ and vertical position $L_{y}$ $=10$ pixels $(136.8 \mu \mathrm{m})$ behind the crack tip. 


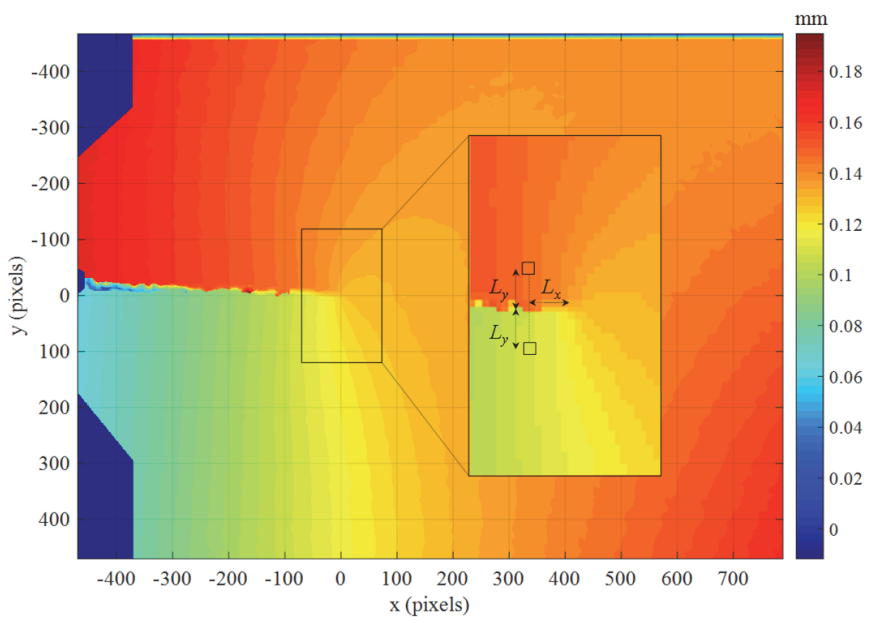

Figure 4: Magnified view of the crack tip region illustrating how CTOD measurements are based on a particular pair of points behind the crack tip. The coordinate axes have been modified with their origin at the crack tip.
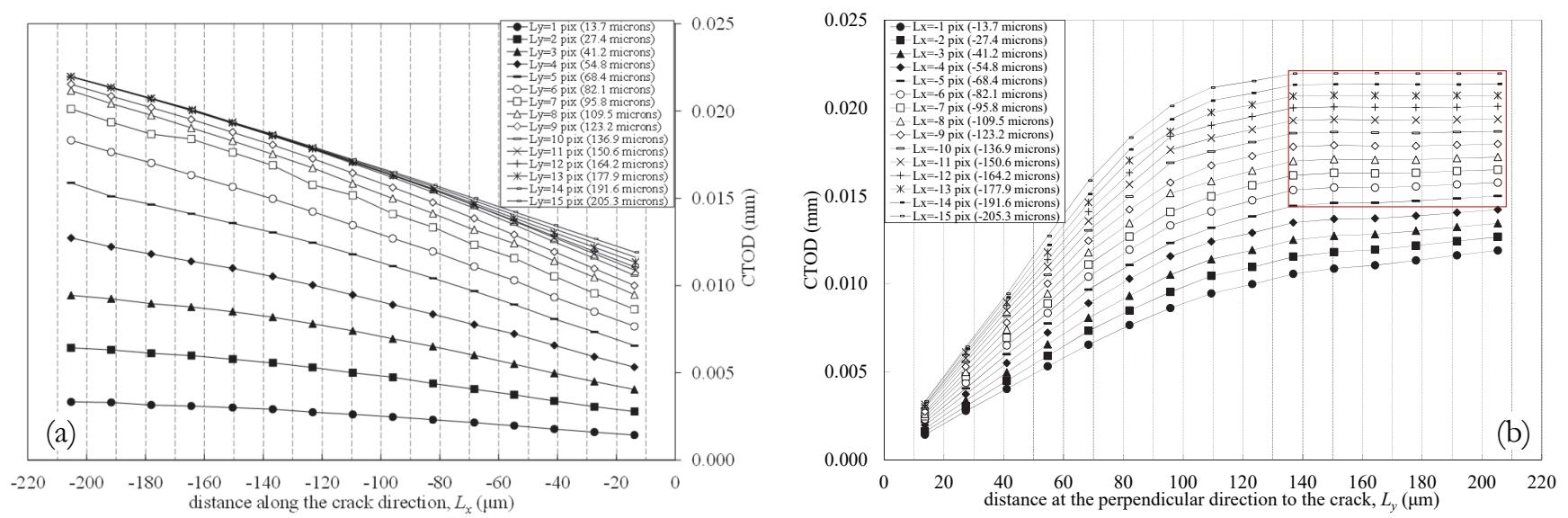

Figure 5: Graphs showing the effect of the location behind the crack tip of the selected points used for the CTOD measurement. (a) As a function of the distance along the crack plane $L_{x}$; (b) As a function of the distance in the perpendicular direction $L_{y}$ to the crack plane.

\section{EXPERIMENTAL RESULTS}

$\mathrm{O}$

nce the values of $L_{x}$ and $L_{y}$ were established, the CTOD through a loading cycle for any particular crack length could be obtained from analysis of the DIC data. A typical plot showing CTOD at increments of $25 \mathrm{~N}$ throughout a full loading cycle for a crack length of $9.40 \mathrm{~mm}$ in the case of the specimen tested at $R=0.1$ and a crack length of $9.20 \mathrm{~mm}$ in the case of the specimen tested at $R=0.6$ is shown in Fig. 6. The elastic and plastic CTOD values can be obtained from the data given in Fig. 6, using an offset procedure similar to that reported by Skorupa et al. [10] for compliance measurements. In this process, a least squares straight line was fitted to experimental data over the part of the load cycle that corresponds with elastic deformation (see lines drawn in Fig. 6). The elastic portions of the loading cycle were identified using a 3 point sliding average, founding a range between 15\% and $55 \%$ of the loading range from the minimum applied load for the specimen tested at $R=0.1$ and between the minimum applied load and a $40 \%$ of the loading range from the minimum load for the specimen tested at $R=0.6$. To evaluate how the components of CTOD can change depending on the loading range selected, a sensitivity analysis was performed to evaluate the errors made when variations of $\pm 10 \%$ for the lower and upper limits are analysed. According to this sensitivity analysis, the variations of the lower limit involved errors between $3-4 \%$ for the plastic CTOD and $1.0-1.5 \%$ for the elastic component. On the other hand, the variations on the upper limit involved errors between $6.5-10 \%$ for the plastic CTOD and 3-4.5\% for the elastic CTOD. From these results it is evident that the selection of the upper limit is more restrictive than the lower limit. This is 
not strange since the upper limit establishes the separation between the elastic and plastic components. Therefore, these results obtained from the sensitivity analysis strengthen the definition of the loading range for describing the elastic behaviour. The equation obtained from this linear fit was employed to calculate the theoretical CTOD value corresponding to all analysed load levels. Thus, the CTOD offset was obtained from Eq. (1). The ranges of the elastic and plastic components of the CTOD can then be defined as shown in Fig. 6.

$$
C T O D \text { offset }=\frac{C T O D_{t h}-C T O D_{\text {exp }}}{C T O D_{t h}} \cdot 100
$$



(1)

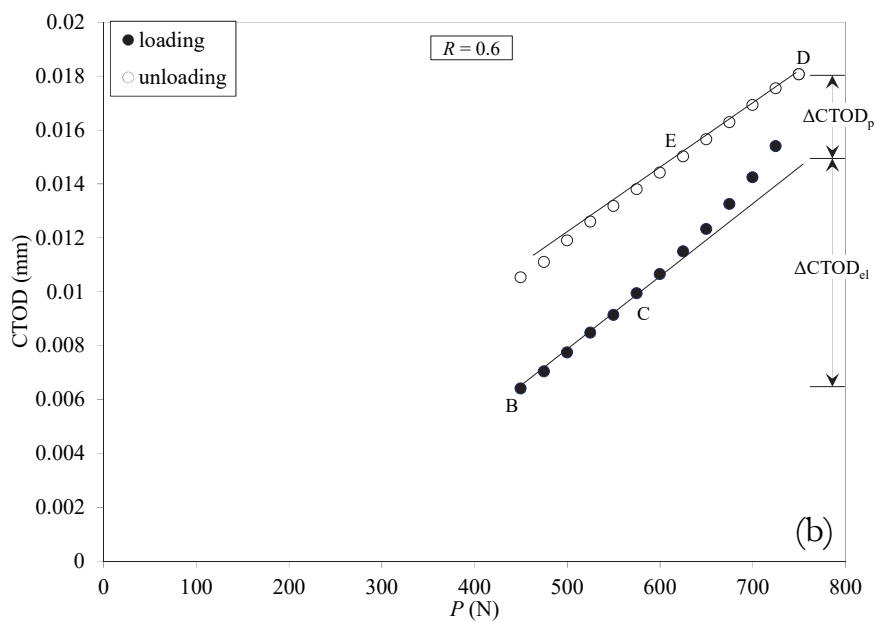

Figure 6: Plot of CTOD through a complete loading cycle, showing its elastic and plastic components at a crack length of $9.40 \mathrm{~mm}$ for the specimen tested at $R=0.1$ (a) and $9.20 \mathrm{~mm}$ for the specimen tested at $R=0.6$ (b) using 5 pixels $(68.4 \mu \mathrm{m})$ and 10 pixels $(136.8$ $\mu \mathrm{m})$ for the distances $L_{x}$ and $L_{y}$, respectively.


Figure 7: Variation of CTOD offset as a function of the applied load at a crack length of $9.40 \mathrm{~mm}$ for the specimen tested at $R=0.1$ (a) and $9.20 \mathrm{~mm}$ for the specimen tested at $R=0.6$ (b). A value of $4 \%$ was set as the offset criterion to define the region of the curve corresponding to the elastic component of the CTOD.

The CTOD offset is plotted against applied load (Fig. 7) and the elastic region is determined in accordance with the standard E 647 [30], where a CTOD change of 4\% either side of the zero value is taken as representing the offset criterion (marked in Fig. 7 and corresponding with a compliance change of $\approx 4 \%$ ) and establishes the elastic region in the offset CTOD trace. The letters $A$ to $D$ in Figs. $6 a$ and $7 \mathrm{a}$ define the various regions in the CTOD loading cycle for the specimen tested at $R=0.1$, where the load range between $B$ and $C(175 \mathrm{~N}$ to $450 \mathrm{~N})$ defines elastic behaviour. It is clear in Fig. $6 a$ that from point $C$ there is a deviation from linearity up to point $D$, corresponding to the maximum applied load, and this is attributed to plastic deformation. The elastic and plastic ranges of CTOD can then be estimated by extrapolating the linear regime to the maximum load (Fig. 6). During unloading, there is a linear decrease in CTOD between points $D$ and 
E with the same slope than that obtained for the elastic regime in the loading half cycle. As is the case for the loading half cycle, there is a change in the linearity during further unloading due to the reversed plastic deformation.

The elastic and plastic ranges of CTOD during the loading half cycle were therefore obtained from Fig. $6 \mathrm{a}$ as $13 \mu \mathrm{m}$ and 6 $\mu \mathrm{m}$, respectively and the corresponding values for the unloading half cycle were $13.2 \mu \mathrm{m}$ and $5.8 \mu \mathrm{m}$, respectively. The plastic CTOD is hence 31.6\% (loading) and 30.5\% (unloading) of the maximum CTOD. The region between points $A$ and $B$ in Figs. $6 a$ and $7 \mathrm{a}$ is associated with crack opening and closing and additional work could be done to estimate the opening and closing loads; however, any additional information gained on the subjects of crack closure and crack tip shielding is both complex in interpretation of its true effects, and is not germane to the present study.

Similar procedure can be extrapolated in the case of the specimen tested at $R=0.6$ but with the difference that the loading range is between $450 \mathrm{~N}$ and $750 \mathrm{~N}$. The elastic and plastic ranges of CTOD were $8.7 \mu \mathrm{m}$ and $2.9 \mu \mathrm{m}$, respectively. It is clear the difference between these values and those obtained for $R=0.1$. However, these values are coherent since the loading range for the test at high ratio is a $44 \%$ of that corresponding to the low ratio. Another important difference respect the CTOD plot at low $\mathrm{R}$-ratio is that the region between $A$ and $B$ point was not identified. This is not surprising since at $\mathrm{R}=0.6$ any crack shielding effect was not expected.

The methodology described above to obtain the CTOD range for the elastic and plastic components was applied to data recorded during crack growth for the tests conducted at the stress ratios of $R=0.1$ and 0.6. Fig. 8a shows the elastic and plastic CTOD data as a function of crack length. Significant scatter is observed in the elastic CTOD values, being those at $R=0.6$ about a half of those values at $R=0.1$; while those representing the plastic CTOD show a more consistent increment with increase in crack length. Plastic CTOD demonstrates a faster rate of increase at $R=0.1$ than at $R=0.6$. However, normalising the elastic and plastic ranges of CTOD values by the total range of CTOD gives a much more uniform picture at the two stress ratio values (Fig. 8b) and the data for the elastic CTOD now shows more consistent behaviour. The trends in the CTOD curves in Fig. 8b appear sensible since, as the crack is propagating, the plastic deformation is increasing and, with it, the plastic CTOD, whilst the value of the elastic CTOD undergoes a corresponding decrease as a percentage of the total CTOD.
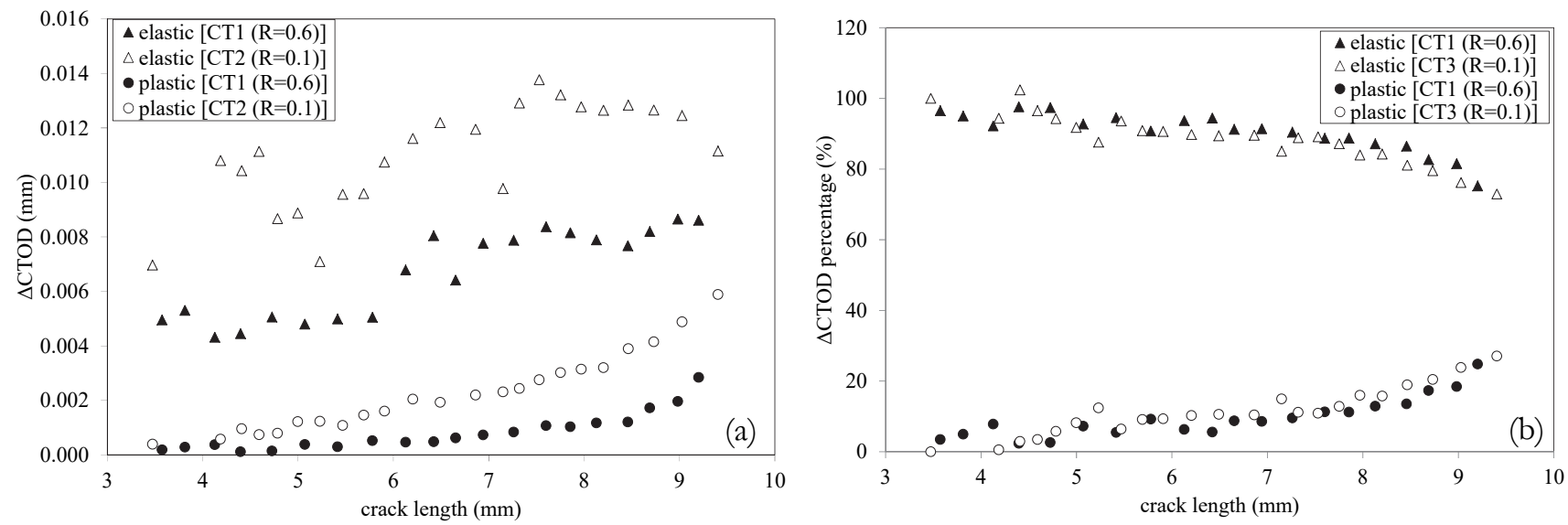

Figure 8: (a) Elastic and plastic CTOD ranges as a function of the crack length for both tests. (b) Elastic and plastic CTOD ranges as a percentage of the total CTOD range as a function of crack length.

Fig. 9a presents data for $d a / d N$ as a function of the ranges of $\mathrm{CTOD}_{t}, \mathrm{CTOD}_{e l}$ and $\mathrm{CTOD}_{p}$ for the specimen tested at $\mathrm{R}$ $=0.1$. It is clear that only the plastic component of CTOD shows a consistent increase with crack growth rate and could

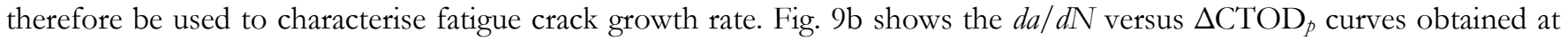
both $R=0.1$ and 0.6 that demonstrate a very good correlation of fatigue crack growth rate into a single linear relationship. It should be noted that this correlation is obtained without employing logarithmic scales as is necessary when using the Paris law. The crack growth rate is then given by linear regression as:

$$
\frac{d a}{d N}=0.2706 \times \Delta C T O D_{p}
$$


The constant in Eq. (2) does not have units, unlike the constants defined by Paris law, and a linear relationship between $d a / d N$ and the range of CTOD was also observed by Guo et al. [11], Tvergaard [20] and Pippan and Grosinger [32]. Those works, however, all used the total CTOD and they did not distinguish between the plastic and elastic CTOD components. A linear relationship between $d a / d N$ and $\Delta \mathrm{CTOD}_{p}$ has been reported by Antunes et al. [33] in work on 7050-T6 aluminium alloy that combined numerical modelling and experimental data in recently published work. They obtained $d a / d N$ data from experiments in middle-tension (MT) specimens for different stress ratios while the plastic CTOD data were numerically determined by using the methodology previously reported in [21]. They obtained the relationship $d a / d N=0.5246 \times \Delta \mathrm{CTOD}_{p}$.

In contrast, in the experimental work presented here, $\mathrm{da} / \mathrm{dN}$ data were obtained from measurements of crack length and number of cycles, with the CTOD data obtained from analysis of DIC measurements of the vertical displacement. To the knowledge of the present authors, this is the first time that plastic CTOD data, determined solely by experimentation, has been used to characterise fatigue crack growth rate at two different stress ratio values.
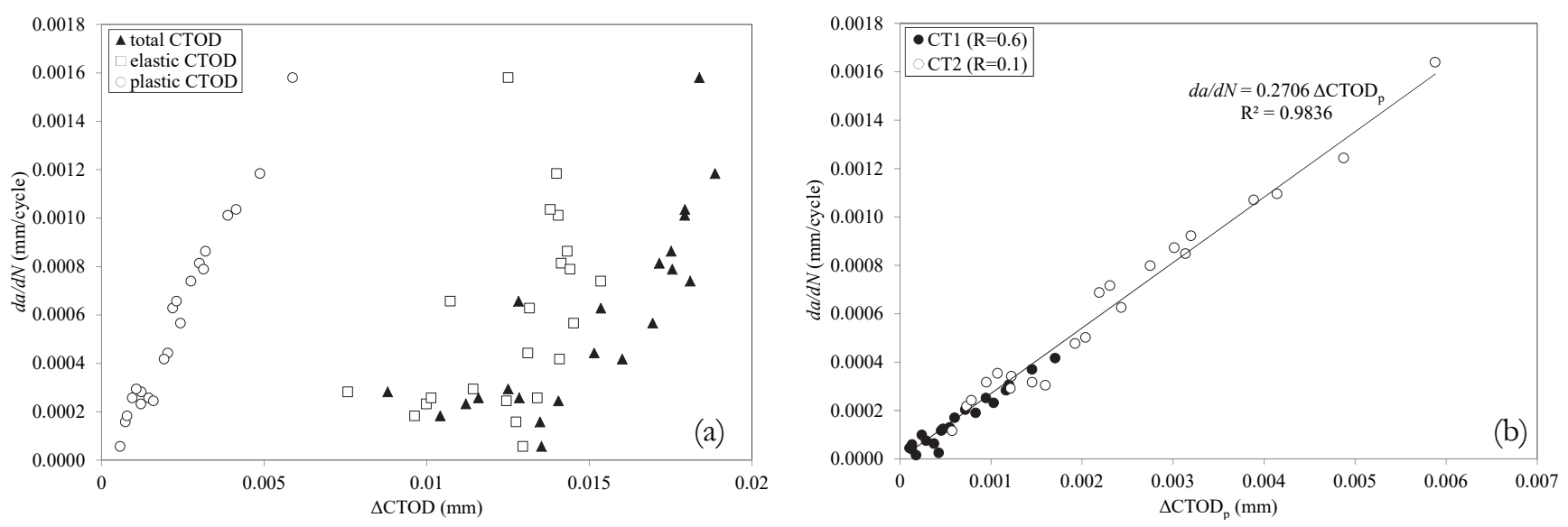

Figure 9: (a) Crack growth rate per cycle $(d a / d N)$ as a function of the range of total CTOD $\left(\Delta C \operatorname{COD}_{t}\right)$, elastic CTOD $\left(\Delta \mathrm{CTOD} \mathrm{C}_{\ell}\right)$ and plastic CTOD $\left(\Delta \mathrm{CTOD}_{p}\right)$ for the specimen tested at low stress ratio $(\mathrm{R}=0.1)$. (b) Plot of $d a / d N$ versus $\Delta C$ TOD $p$ for the two tests conducted at low $(R=0.1)$ and high $(R=0.6)$ stress ratio values.

\section{ConClusions}

he plastic range of CTOD is clearly linked with the plastic deformation generated at the crack tip during fatigue crack propagation at constant amplitude. The range of plastic CTOD ( $\triangle$ CTODp) has therefore been used to characterise and correlate fatigue crack growth data obtained at two different stress ratio values of 0.1 and 0.6 . A linear relationship between $\mathrm{da} / \mathrm{dN}$ and $\triangle$ CTODp, independent of stress ratio was, observed for a CP titanium alloy. The CTOD was measured from the relative vertical displacement between the crack flanks. A sensitivity analysis was performed to determine the optimum position behind the crack tip of the points where displacement was measured, and it was found that the CTOD value showed a significant dependence on their location in the plane of the crack. However, the influence of the vertical distance from the crack plane was not as restrictive, with a stable value of CTOD being obtained at a distance of $136.9 \mu \mathrm{m}$. This conclusion was reached using a methodology that analysed different displacement profiles plotted from the vertical displacement map.

This work has demonstrated that CTOD represents a viable alternative technique to stress intensity factor in characterising fatigue crack growth rate since CTOD considers fatigue threshold and crack shielding in an intrinsic way [33]. However, further work is necessary to determine whether the linear relationship observed between $d a / d N$ and $\Delta \mathrm{CTOD}_{p}$ may be considered as an intrinsic material property, independent of the geometry and loading conditions. The plastic CTOD approach is, however, unlikely to shed light on the physical mechanisms underlying such phenomena as plasticity-induced crack tip shielding and a combination of approaches will be required to advance understanding, e.g. the use of plastic CTOD and the CJP model of crack tip fields. 


\section{ACKNOWLEDGEMENTS}

7 he current work has been conducted with the financial support from Gobierno de España through the project 'Proyecto de Investigación de Excelencia del Ministerio de Economía y Competitividad MAT2016-76951-C2-1-P'.

\section{REFERENCES}

[1] Paris, P.C. (1960). A critical analysis of crack propagation laws, J. Basic Eng., 85, pp. 528-534.

[2] Wells, A.A. (1961). Unstable crack propagation in metals. Cleavage and fast fracture, Proceedings of the Crack Propagation Symposium, Cranfield, UK, 1, 84.

[3] Laird, C., Smith, G.C. (1962). Crack propagation in high stress fatigue, Philos. Mag. 8, pp. 847-857.

[4] Vasco-Olmo, J.M., Díaz, F.A., García-Collado, A., Dorado, R. (2013). Experimental evaluation of crack shielding during fatigue crack growth using digital image correlation, Fatigue Fract. Engng Mater. Struct., 38, pp. $223-237$.

[5] Antunes, F.V., Branco, R., Costa, J.D., Rodrigues, D.M. (2010). Plasticity induced crack closure in middle-tension specimen: numerical versus experimental, Fatigue Fract. Engng Mater. Struct. 33, pp. 673-686.

[6] Chu, T.C., Ranson, W.F., Sutton, M.A., Peters, W.H. (1985). Applications of digital-image correlation technique to experimental mechanics, Exp. Mech., 25, pp. 232-244.

[7] De Matos, P.F.P., Nowell, D. (2007). On the accurate assessment of crack opening and closing stresses in plasticityinduced fatigue crack closure problems, Eng. Fract. Mech., 74, pp. 1579-1601.

[8] Antunes, F.V., Rodrigues, S.M., Camas, D. (2016). A numerical analysis of CTOD in constant amplitude fatigue crack growth, Theoretical and Applied Fracture Mechanics, 85, pp. 45-55.

[9] Korsunsky, A.M., Song, X., Belnoue, J., Jun, T., Hofmann, F., De Matos, P.F.P., Nowell, D., Dini, D. (2009). Aparicio-Blanco, O., Walsh, M.J., Crack tip deformation fields and fatigue crack growth rates in Ti-6Al-4V, Int. J. Fatigue, 31, pp. 1771-1779.

[10] Skorupa, M., Beretta, S., Carboni, M., Machniewicz (2002). An algorithm for evaluating crack closure from local compliance measurements, Fatigue Fract. Engng Mater. Struct., 25, pp. 261-273.

[11] Guo, W., Wang, C.H., Rose, R.F. (1999). The influence of cross-sectional thickness on fatigue crack growth, Fatigue Fract. Engng Mater. Struct., 22, pp. 437-444.

[12] Tvergaard, V., (2004). On fatigue crack growth in ductile materials by crack-tip blunting, J. Mech. Phys. Solids, 52, pp. 2149-2166.

[13] Pippan, R.G., Grosinger, W., (2013). Fatigue crack closure: From LCF to small scale yielding, Int. J. Fatigue, 46, pp. 41-48. 